\title{
PURE CHITOSAN AND CHITSOAN/CHITOSAN LACTATE BLENDED NANOFIBRES MADE BY SINGLE STEP ELECTROSPINNING
}

\author{
Tong Cheng ${ }^{1 *}$, Rolf-Dieter Hund ${ }^{1}$, Dilibaier Aibibu ${ }^{1}$, Jana Horakova², Chokri Cherif ${ }^{1}$
}

${ }^{1}$ Institute of Textile Machinery and High Performance Material Technology (ITM), Technische Universität Dresden, Hohestrasse 6, 01069, Dresden, Germany ${ }^{2}$ Faculty of Textile Engineering Department of Nonwovens and Nanofibrous Materials Technical University of Liberec (TUL) Studentska 2, 46117, Liberec, Czech Republic e-mail: cheng@itb.mw.tu-dresden.de

\begin{abstract}
:
A single step electrospinning of chitosan and chitosan derivative-chitosan lactate nanofibres was studied in this paper. Chitosan was dissolved into acetic acid to produce structure-stable nanofibres. The effect of chitosan concentration and the content of acetic acid on the fibre diameter and morphology of nanofibres were studied in detail. The dynamic viscosity and surface tension of the electrospinning chitosan solutions were systematically studied as well. Based on the fundamental study on electrospinning chitosan in acetic acid, a chitosan derivative, chitosan lactate, was added to produce nanofibre in a $\mathrm{pH}$-friendly aqueous environment. Chemical and morphological analyses demonstrated that chitosan lactate will positively influence the formation of nanofibres in higher $\mathrm{pH}$ condition although the morphology should be improved.
\end{abstract}

\section{Keywords:}

Electrospinning, chitosan, chitosan lactate, nanofibre, surface tension

\section{Introduction}

Nowadays, the development of chitosan, one of the prospective biomaterials with various applications in bio-areas, arouses much attention. The main interests of several research groups are with a broad range of applications, such as filtration [1], antimicrobial [2], biosensor [3], drug delivery systems [4-6], wound dressing $[7,8]$ and tissue engineering $[9,10]$.

Chitosan is a derivate of chitin, the second most abundant natural polysaccharide after cellulose, synthesised by a number of living organisms [11]. Because chitin cannot be dissolved in common solvents, the application of this type of material should be focused on its deacetylated derivate, chitosan. It is observed that chitosan is a copolymer of $\mathrm{N}$-acetyl-D-glucosamine and D-glucosamine. The degree of deacetylation (DDA) of chitin to chitosan determines the content of D-glucosamine in chitosan. The $\beta-1$, 4-linked-D-glucosamine attached on the sugar background of chitosan shows a structure of chitosan similar to cellulose except for the amino group that replaces the hydroxyl group on the C-2 position [12]. Chitosan displays good biocompatibility, hydrophilicity, immunogenicity, biodegradability and bioresorbable degradation [13,14]. All these give chitosan a promising reputation for its welcome applications in different areas.

One of the popular researches with chitosan is electrospinning nanofibres of this material. Electrospinning is considered a novel method for spinning polymers as extremely fine fibres. The principle of an electrospinning polymer-solvent requires the polymer to be electrically charged to a fluid jet. This fluid jet is formed when the polymer droplet acts as a viscous force which is high enough to overcome the forces of the surface tension. The fluid jet will be collected on a piece of metal (collector) stationed at a distance of over $3 \mathrm{~cm}$. Over this travelling distance, the solvent evaporates and forms fibres on the collector. Meanwhile, the viscosity of the solution for the polymer acts as another critical parameter [15].

However, electrospinning of natural polymers like chitosan is a complex process due to its polycationic specification, molecular weight and wide molecular weight distribution [16]. Most research groups had electrospun chitosan with other polymers, such as chitosan/polyethylene oxide [17], chitosan/ polycaprolacton [18], chitosan/polyvinyl alcohol [19] and chitosan/polyethylene terephthalate [20]. Electrospinning of pure chitosan was hindered by its limited solubility in aqueous acids and the high degree of inter- and intrachain hydrogen bonding [1]. The most common solvent for electrospinning chitosan, especially with uniform fibre formation, is acetic acid and trifluroacetic acid (TFA). Although TFA is not environmentally harmful and toxic solvent, the fibre electrospun from TFA readily dissolved in aqueous media and had to be cross-linked to preserve its structural integrity [21]. When using acetic acid as solvents, the concentration of acetic acid is always very high which leads to the difficulty of directly applying the chitosan electrospinning products used in bio-application, especially with cell cultivation.

The aim of this work is focused on increasing the $\mathrm{pH}$ value of electrospinning condition to produce neutral electrospinning nanofibres. Chitosan lactate is chosen in our work because chitosan lactate is a water soluble chitosan derivative and lactate is the salt of an organic acid commonly present in the human body, which has been demonstrated to be biocompatible and biodegradable in either drug or gene delivery systems [22]. In this work, pure chitosan and chitosan/ chitosan lactate blended nanofibres are produced by a single step electrospinning technology with high $\mathrm{pH}$ condition in which the final chitosan products are beneficial for bio-application. 
Bead-free nanofibre mats will be achieved by optimising the electrospinning process. An aqueous acetic acid was chosen in this work because of its good reputation to dissolve chitosan as a monobasic organic acid. A systematic study was performed to understand the effect of the acetic acid concentration, the content of chitosan and solution viscosity on the nanofibre producing, especially the morphology of the fibre. Furthermore, a type of chitosan derivative, chitosan lactate, was applied to electrospin the nanofibres.

\section{Experimental}

\subsection{Materials}

Chitosan (95/500) and chitosan lactate were kindly provided by Heppe Medical Chitosan, Germany. Acetic acid was purchased from MERCK, Germany. Water was deionised.

\subsection{Solution preparation}

Chitosan was dissolved into acetic acid with concentration of $3 \%$ and the solutions were stirred for 24 hours at room temperature. $50 \%, 60 \%, 70 \%, 80 \%$ And $90 \%$ acetic acid was used to prepare electrospinning Chitosan solutions.

Chitosan lactate was dissolved in water and different concentrations of acetic acid for electrospinning as well. A blend of chitosan and chitosan lactate (ratio 1:1) was prepared in acetic acid separately. The chitosan (3\% in $10 \%$ of acetic acid) was blended with chitosan lactate (3\% in $10 \%$ of acetic acid) with a consistent stirring for 24 hours at room temperature.

The final chitosan/chitosan lactate solutions were filtered through PTFE membranes with pore sizes of $100 \mu \mathrm{m}$ and immediately used for electrospinning.

\subsection{Electrospinning}

The solution was placed into a $3 \mathrm{ml}$ syringe with a fitted needle. The inner diameter of the needle was $0.4 \mathrm{~mm}$. A copper wire connected to the positive electrode was attached to the spinning needle. The collector was grounded and covered with an aluminium foil. A high voltage power supplier was employed to generate electric fields up to $30 \mathrm{kV}$ between the spinning needle and the collector. Electrospinning chitosan nanofibre was conducted by applying a voltage of $30 \mathrm{kV}$. A syringe pump (Q) gave the solution a rate between 0.1 and $1 \mathrm{ml} / \mathrm{h}$. The distance (d) from the tip to the collector was $10 \mathrm{~cm}$. The spun nanofibres were deposited on the collector. The electrospinning process was performed at room temperature.

\subsection{Characterisation}

\subsubsection{SEM}

The surface morphology of nanofibres was analysed by a scanning electron microscope (SEM) Phenom Pro (PhenomWorld, Netherlands). The samples were sputtered with gold before analysing. A total of 100 fibres were measured using the image analysis software NIS Elements 3.0 to get the average fibre diameters.

\subsubsection{Viscosity measurement}

The dynamic viscosity of chitosan and chitosan derivative solutions were measured by a HAAKE MARS Modular Advanced Rheometer System (Thermo Scientific, Germany).

\subsubsection{Surface energy measurement}

The dynamic surface energy of chitosan and chitosan derivative solutions were measured by Single Fibre Tensiometer K100SF (KRÜSS, Germany).

\subsubsection{FTIR}

Infrared measurements were performed on a Nicolet 6700 (Thermo Scientific, Germany) Fourier-transform infrared spectroscopy (FTIR). The samples were pressed onto a diamond crystal and 64 scans were averaged for a spectrum. Scans were taken from $650 \mathrm{~cm}-1$ to $4000 \mathrm{~cm}-1$ with a resolution of $4 \mathrm{~cm}-1$. Background was taken right with no sample on the crystal before every measurement, also averaged over 64 scans.

\section{Results and Discussion}

\subsection{Electrospinning of chitosan}

The initial experiments are focused on electrospinning of pure chitosan with $95 \%$ DDA and molecular weight of Mw=900 kDa. Table 1 summarised the results of electrospun nanofibre from different chitosan solutions. In order to analyse the effect of acetic acid concentration on nanofibre formation, the concentration of acetic acid ranged from $10 \%$ to $50 \%-90 \%$ with

Table 1. Results of electrospun chitosan nanofibre with different concentrations of acetic acid and different content of chitosan

\begin{tabular}{|c|c|c|c|c|}
\hline & $1 \%$ chitosan & $2 \%$ chitosan & $3 \%$ chitosan & $4 \%$ chitosan \\
\hline $10 \%$ acetic acid & beads or $x x^{*}$ & beads or $\mathrm{xxx}$ & beads or $x x x$ & beads or $\mathrm{xxx}$ \\
\hline $50 \%$ acetic acid & beads & beads & beads+fibres & $x x x$ \\
\hline $60 \%$ acetic acid & beads & beads & beads+fibres & $x x x$ \\
\hline $70 \%$ acetic acid & beads & beads & beads+fibres & fibres \\
\hline $80 \%$ acetic acid & beads & beads+fibres & fibres & fibres \\
\hline $90 \%$ acetic acid & beads+fibres & beads+fibres & fibres & fibres \\
\hline
\end{tabular}

\footnotetext{
${ }^{*} x x x$ means that no fibre was formed
} 
the difference of $10 \%$ for each. $1 \%, 2 \%, 3 \%$ And $4 \%$ chitosan solutions were used for each concentration of acetic acid.

Figure 1 shows the morphology of chitosan nanofibres which were produced from different concentrations of acetic acid aqueous solution and from a $3 \%$ content of chitosan. Chitosan that was dissolved in acetic acid with a concentration of $10 \%$ did not result in any fibre with varying concentration of chitosan (Figure 1a). With increasing concentration of acetic acid, the morphology of the nanofibres has changed from beads to a fibre structure. When the concentration of acetic acid is higher than $50 \%$, a blended structure of beads and fibres was obtained. The best result was achieved with $90 \%$ acetic acid which results in uniform and bead-free nanofibres.

The structural change from beads to fibres of nanofibre with increasing concentration of acetic acid can be explained by surface energy impact. As shown in Figure 1b, electrospun chitosan in lower concentration of acetic acid was captured. The high surface tension of the solution inhibits the electrospinning process because of the instability of the jets and the generation of sprayed droplets. An attempted fibre could be seen between two particles (Figure 1b). Figure 2 shows the dynamic surface tension of $3 \%$ chitosan solutions with different concentrations of acetic acid. The main values of surface tension of solutions are listed in Table 2. With higher concentrations of acetic acid, the surface tension of chitosan solution decreased. It indicates that, when the polymer exceeds a certain surface tension, the fibre could be elongated. A concentration of $90 \%$ acetic acid has the lowest surface tension $(30.76 \mathrm{mN} / \mathrm{m})$ and is a most suitable solvent for electrospinning of bead-free uniform chitosan nanofibres (Figure 1d).
Table 2. Surface tension of chitosan solutions with different concentrations of acetic acid

\begin{tabular}{|c|c|}
\hline Solution & $\begin{array}{c}\text { Surface tension } \\
(\mathbf{m N} / \mathbf{m})\end{array}$ \\
\hline $\mathbf{3 \%}$ Chitosan in $\mathbf{1 0 \%}$ acetic acid & 43.70 \\
\hline $\mathbf{3 \%}$ Chitosan in $\mathbf{5 0 \%}$ acetic acid & 33.88 \\
\hline $\mathbf{3 \%}$ Chitosan in $\mathbf{8 0} \%$ acetic acid & 31.52 \\
\hline $\mathbf{3 \%}$ Chitosan in $\mathbf{9 0 \%}$ acetic acid & 30.76 \\
\hline
\end{tabular}

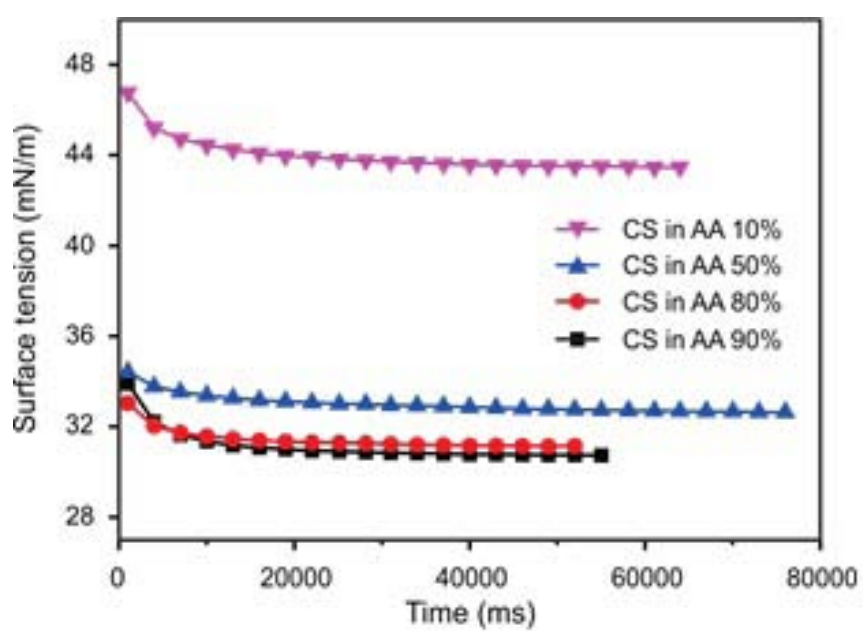

Figure 2. Dynamic surface tension of $3 \%$ chitosan (CS) solutions with different concentrations of acetic acid (AA)
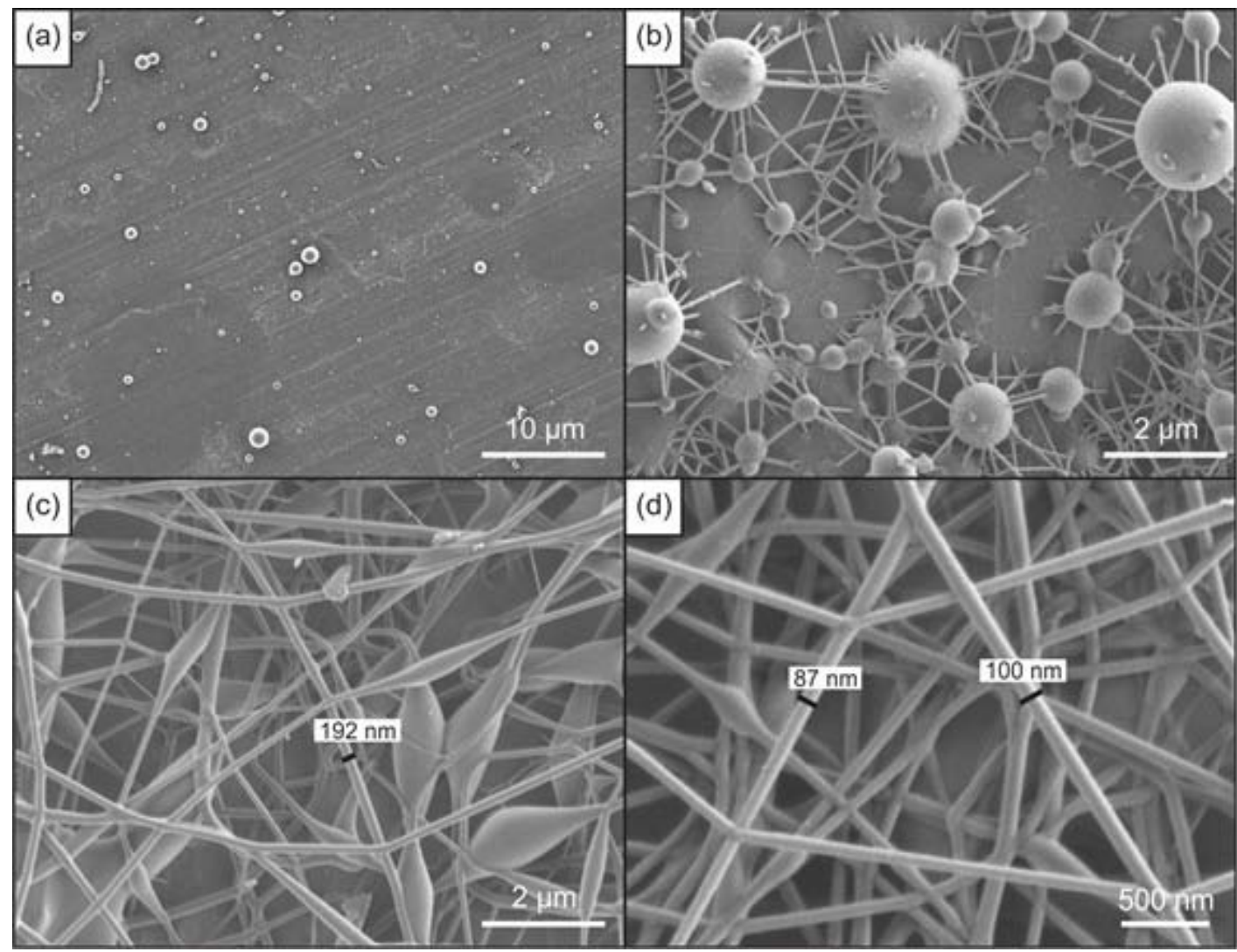

Figure 1. SEM results of chitosan nanofibre by the condition of $3 \%$ chitosan dissolved into different concentrations ((10 \% (a); $50 \%$ (b); $80 \%$ (c); $90 \%(d))$ of acetic acid 
Different concentrations of chitosan solutions were electrospun to determine the optimum concentration for producing uniform bead-free nanofibres. At high chitosan concentration, fibres with larger diameters were produced. For example, with $1 \%$ chitosan, which is a very low electrospinning concentration, beads were deposited on the collector. A $2 \%$ chitosan solution offers a combination of beads and fibres. When $3 \%$ and $4 \%$ chitosan solutions were used, uniform fibres were produced. The maximum concentration for electrospinning with chitosan (95/500) was $4 \%$ chitosan solution. Higher concentrations result in a gel which was not electrospinnable. The strong hydrogen bonding between $-\mathrm{NH}_{2}$ and $-\mathrm{OH}$ groups of chitosan molecules will increase with higher concentration of chitosan in acetic acid aqueous solution and yields an unspinnable result.

Apart from the morphology of the fibres, using different concentrations of chitosan also contributes to the nanofibre diameter. The fibre diameters were about $80 \mathrm{~nm}$ in all cases. Slight increases of fibre diameters were observed with increased concentration of the solution. Within $90 \%$ acetic acid aqueous solution and $2 \%$ chitosan, average fibre diameters of $61.40 \pm 8.75 \mathrm{~nm}$ (Figure 3a) can be realised; 3\% chitosan 76.87 $\pm 19.55 \mathrm{~nm}$ and $4 \%$ chitosan had average fibre diameters of $78.83 \pm 19.39 \mathrm{~nm}$ (Figure $3 \mathrm{~b}$ and $\mathrm{c}$ ).

The viscosity of chitosan solutions was measured to analyse their influence on nanofibre formation and on fibre morphology. Table 3 summarises the dynamic viscosities of chitosan solutions which were used for electrospinning. It has been found that low viscosity in the solution for electrospinning gave beads instead of continuous fibres (Figure 1b). At low concentrations, where the viscosity of solution is low while surface tension is relatively high, the solution jet could not maintain its own shape at the end of the tip and form a small drop among the fibres. At higher concentrations, the electrospinning process could be hindered by an inability to control and maintain the flow of a polymer solution to the tip of the needle and by the cohesive nature of the high-viscosity solutions.

\subsection{Electrospinning of chitosan derivative}

The bead-free chitosan nanofibres in single-step electrospinning have been successfully developed. However, aqueous acetic acid solutions can only dissolve a very small amount of chitosan, which means that the chitosan fibre could only be obtained by electrospinning with a high concentration of acetic acid ( $90 \%$ as discussed above). Such an excess acid condition may not be desirable for bio-application, especially for an acid-labile drug system [22]. In order to avoid the high acid environment, the second part of the work focused on adding chitosan lactate derivative into chitosan to reduce the concentration of acetic acid.

The FTIR results of chitosan and chitosan lactate are shown in Figure 4. Comparing the FTIR results of both solutions, all spectra exhibited broad peaks in the range of $3450-3400 \mathrm{~cm}^{-1}$. The peaks were assigned to $\mathrm{OH}$ stretching, indicating intermolecular hydrogen bonding. The spectra also overlapped in the same region of an $\mathrm{NH}$ stretching. The $\mathrm{C}=\mathrm{O}$ stretching (amide I) peak near $1644 \mathrm{~cm}^{-1}$ representing the structure of

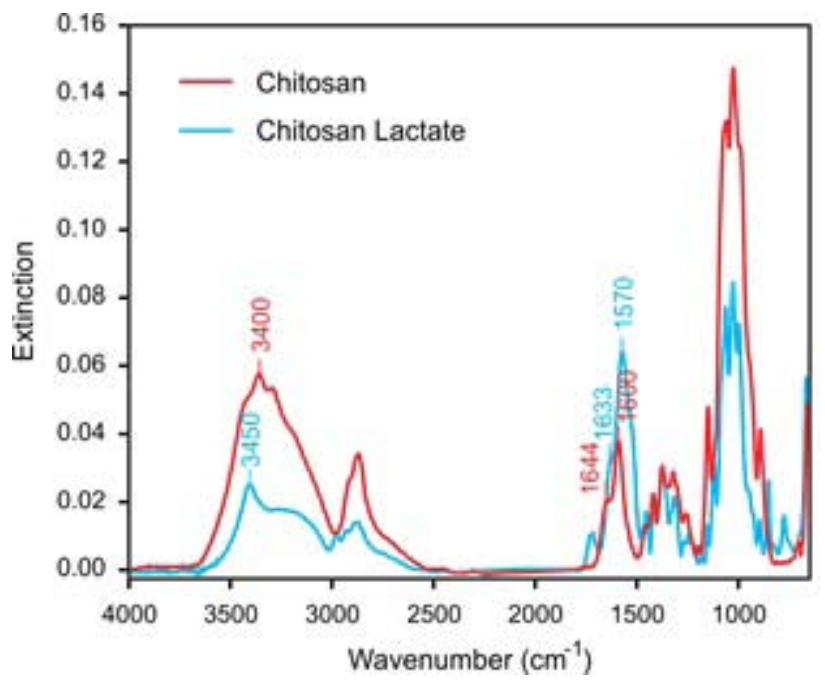

Figure 4. FTIR of chitosan and chitosan lactate

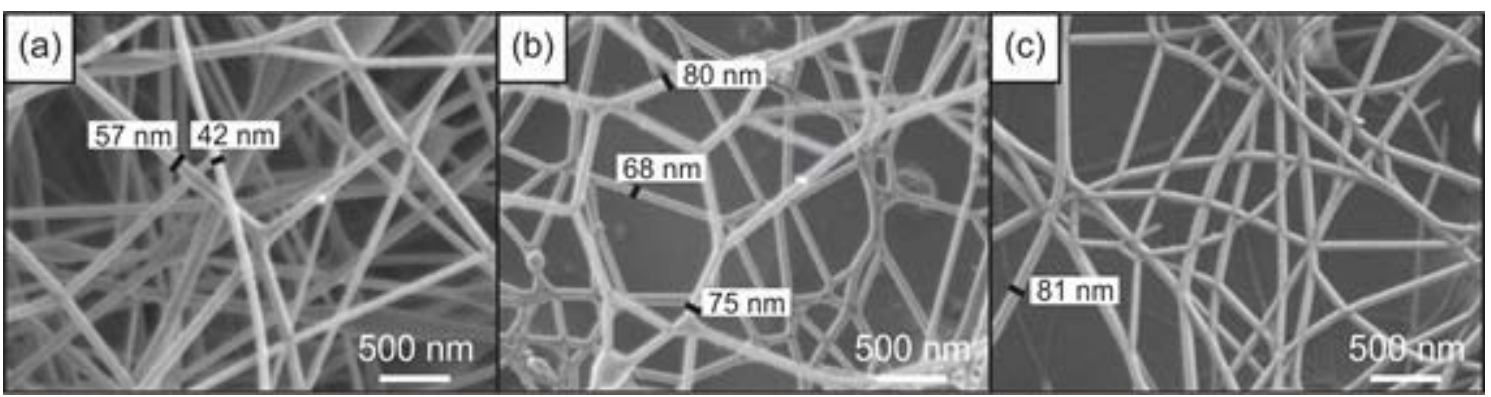

Figure 3. SEM results of variation of chitosan nanofibre diameters by the condition of different concentrations of chitosan dissolved into $90 \%$ acetic acid ((2\% (a); $3 \%(b) ; 4 \%$ (c))

Table 3. Dynamic viscosity of chitosan-acetic acid solutions $(\mathrm{AcOH})$ in $[\mathrm{Pa}$ s] with shear rate of 100

\begin{tabular}{|c|c|c|c|c|c|}
\hline Chitosan & $\mathbf{5 0} \% \mathbf{A c O H}$ & $\mathbf{6 0} \% \mathbf{A c O H}$ & $\mathbf{7 0} \% \mathbf{A c O H}$ & $\mathbf{8 0} \mathbf{A c O H}$ & $\mathbf{9 0} \% \mathbf{A c O H}$ \\
\hline $\mathbf{1} \%$ & 1.0 & 0.4 & 0.4 & 1.2 & 1.3 \\
\hline $\mathbf{2} \%$ & 2.6 & 4.9 & 3.5 & 2.4 & 1.6 \\
\hline $\mathbf{3} \%$ & 8.4 & 15.6 & 8.5 & 10.8 & 8.0 \\
\hline $\mathbf{4} \%$ & 42.5 & 35.3 & 23.7 & 31.5 & 24.0 \\
\hline
\end{tabular}


$\mathrm{N}$-acetylglucosamine as well as the $\mathrm{NH}_{2}$ stretching peak at $1600 \mathrm{~cm}^{-1}$ displaying the glucosamine functional group was exhibited in the spectrum of chitosan. Meanwhile, in Figure 4 (blue curve), the characteristic absorption bands at $1633 \mathrm{~cm}^{-1}$ and $1570 \mathrm{~cm}^{-1}$ are attributed to amide and protonated amino groups of the chitosan lactate.

As conclusion, with a lower polymer concentration, the solution does not contain sufficient material to produce stable solid fibres. For the electrospinning of chitosan lactate, 3\% was chosen. The concentration of acetic acid was controlled between $10 \%$ and $20 \%$ according to the required $\mathrm{pH}$.

The lactate groups in the chitosan derivative lead to hugely different results compared with electrospun chitosan nanofibres. As the chitosan lactate can be dissolved in water, the $3 \%$ of chitosan lactate in water was electrospun directly. Figure $5 a$ shows that no fibre can be found in the SEM results. In the following process, acetic acid was added into the solution and the $\mathrm{pH}$ value was controlled. Chitosan lactate nanoparticles with diameter of $428 \mathrm{~nm}$ (Figure $5 \mathrm{~b}$ ) were formed at $\mathrm{pH} 6.5$. When the $\mathrm{pH}$ of the solution decreased to 5.8 , the particles were still formed. However, they lined up with fibre orientation (Figure 5c). Chitosan lactate can be easily dissolved in water, but it is difficult to obtain uniform chitosan lactate fibres with higher $\mathrm{pH}$ value.

The results of electrospinning of chitosan and chitosan lactate blended nanofibres are illustrated in Figure 5. When the $\mathrm{pH}$ was 6.8 , the thin fibres with uniform morphology and beads were obtained as shown in Figure $5 \mathrm{~d}$. With $\mathrm{pH}$ decreased to 6.0 , the fibres with the big nanoparticles accompanied were observed (Figure 5e). The fibre shows an even, well-formed morphology at pH 5.2 (Figure 5f).

The chitosan lactate dissolved in water exhibits the highest surface tension value of $41.46 \mathrm{mN} / \mathrm{m}$ (Figure 6 and Table 4). When chitosan lactate was dissolved in $10 \%$ of acetic acid, the surface tension reduced to $39.63 \mathrm{mN} / \mathrm{m}$ which was even

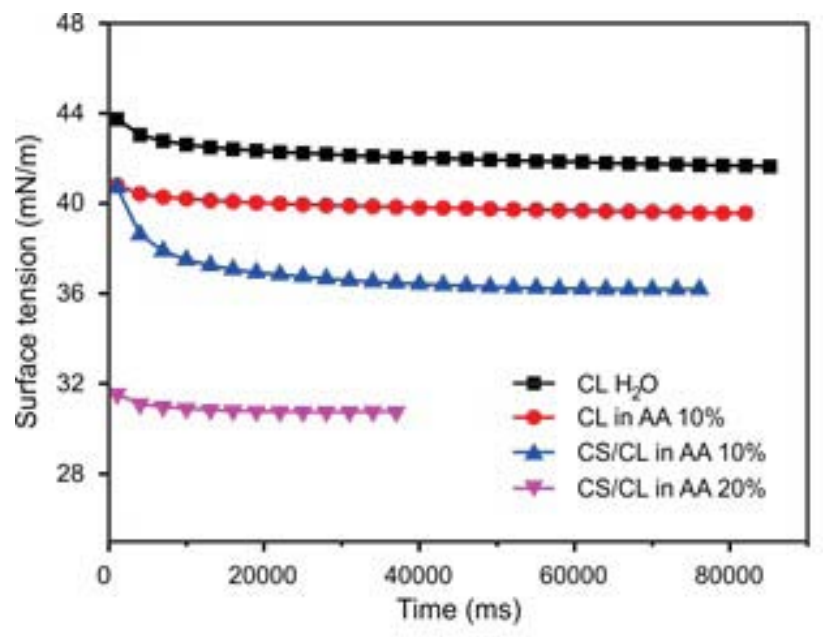

Figure 6. Dynamic surface tension of chitosan lactate $(\mathrm{CL})$ and chitosan/chitosan lactate $(\mathrm{CS} / \mathrm{CL})$ blended solutions

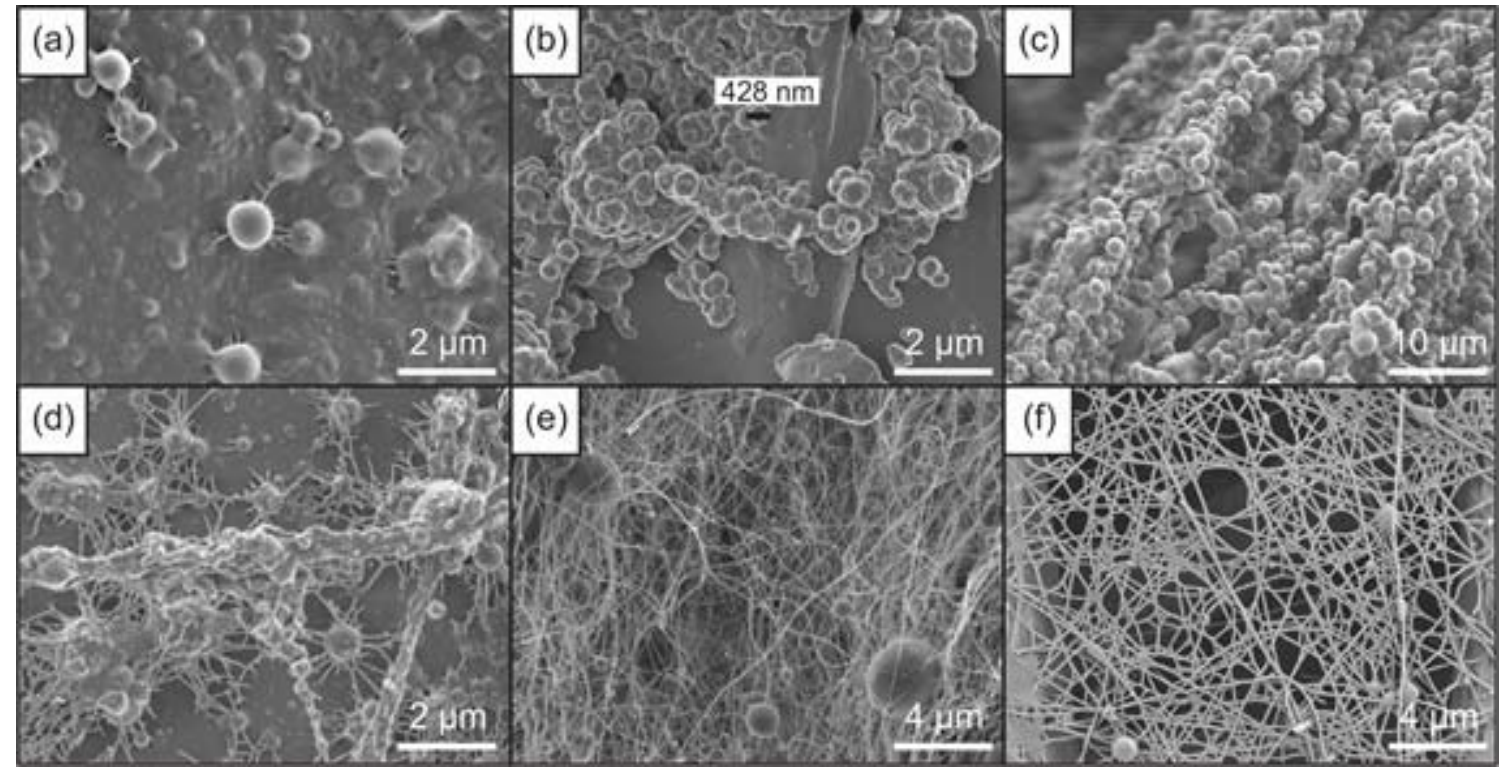

Figure 5. Electrospinning of pure chitosan lactate $(\mathrm{pH}=7$ (a); $\mathrm{pH}=6.5(\mathrm{~b}) ; \mathrm{pH}=5.8(\mathrm{c}))$ and chitosan/chitosan lactate blended $(\mathrm{pH}=6.8(\mathrm{~d}) ; \mathrm{pH}=6.0$ $(\mathrm{e}) ; \mathrm{pH}=5.2(\mathrm{f}))$ in different $\mathrm{pH}$ values

Table 4. Surface tension of chitosan lactate solutions

\begin{tabular}{|l|c|}
\hline Solution & Surface tension $(\mathrm{mN} / \mathrm{m})$ \\
\hline 3\% Chitosan lactate in $\mathrm{H}_{2} \mathrm{O}$ & 41.46 \\
\hline 3\% Chitosan lactate in 10\% acetic acid & 39.63 \\
\hline 3\% Chitosan/chitosan lactate (1:1) in 10\% acetic acid & 36.62 \\
\hline 3\% Chitosan/chitosan lactate (1:1) in 20\% acetic acid & 30.77 \\
\hline
\end{tabular}


lower than the pure chitosan dissolved in the same amount of acetic acid $(43.70 \mathrm{mN} / \mathrm{m}$; Table 2$)$. In the chitosan chain, the $-\mathrm{NH}_{2}$ and $-\mathrm{OH}$ groups of chitosan could form a intramolecular bonding; when the intramolecular force is balanced, the fibre could be formed only in higher concentration of acetic acid. However, in chitosan lactate, the $-\mathrm{NH}_{2}$ was replaced by $\mathrm{NH}_{3}{ }^{+} \mathrm{OOCCH}(\mathrm{OH}) \mathrm{CH}_{3}$ which means the $-\mathrm{NH}_{2}$ groups were in protoned condition. It reduces the intramolecule bonding of $\mathrm{NH}_{2}$ and $-\mathrm{OH}$ groups. When the chitosan lactate was blended with chitosan, the concentration of $\mathrm{NH}_{3}{ }^{+} \mathrm{OOCCH}(\mathrm{OH}) \mathrm{CH}_{3}$ could be increased so that the intramolecule bonding between $-\mathrm{NH}_{2}$ and $-\mathrm{OH}$ groups is decreased. The result is a polymer solution with a lower surface tension. For example, when the ratio of chitosan:chitosan lactate was $1: 1$, and dissolved with $20 \%$ of acetic acid, the surface tension dropped to $30.77 \mathrm{mN} / \mathrm{m}$, which was similar to chitosan solution dissolved in $90 \%$ of acetic acid. As a result, bead-free or particle-free fibre morphology could easily be formed even at a lower concentration of acetic acid $(\mathrm{pH} 5.2)$ as shown in Figure $5 f$.

\section{Conclusions}

The physical and chemical parameters of chitosan solution, such as concentration of acetic acid, chitosan content, viscosity and surface tension, as well as the blend with chitosan lactate can affect the formation and morphology of electrospun nanofibres. A uniformed bead-free nanofibre could be obtained with a higher concentration of acetate acid. The best fibre morphology was achieved by $3 \%$ and $4 \%$ chitosan in $90 \%$ acetic acid. Meanwhile, with higher concentration of chitosan and lower surface tension of the solution, a structurestable and uniform nanofibre can be realised. Chitsoan and chitosan lactate blended solution contain a lower surface tension and a stable electrospinning process to produce beadfree nanofibres in higher $\mathrm{pH}$ condition ( $\mathrm{pH}$ 5.2). The developed pure chitosan nanofibres with higher $\mathrm{pH}$ condition and lower acetic acid concentration will be applied in a further research work in combination with a net-shaped nonwoven scaffold as a hybrid scaffold (nano and micro) for bone tissue-engineering.

\section{Acknowledgements}

This research project (DFG CH 174-24/1) is supported by "Deutsche Forschungsgemeinschaft".

\section{References}

[1] Desai, K., Kit, K., Li, J., Davidson, P. M., Zivanovic, S. and Meyer, H. Polymer. 2009, 50, 3661.

[2] Spasova, M., Paneva, D., Manolova, N., Radenkov, P. and Rashkov, I. Macromolecular Bioscience. 2008, 8, 153.

[3] Huang, X. J., Ge, D. and Xu, Z. K. Eur Polym J. 2007, 43, 3710.

[4] Jiang, H., Fang, D., Hsiao, B., Chu, B. and Chen, W. J Biomater Sci Polym. 2004, 15, 279.

[5] Lee, K. Y., Jeong, L., Kang, Y. O., Lee, S. J. and Park, W. H. Advanced Drug Delivery Reviews. 2009, 61, 1020.

[6] Zhang, Y. Z., Venugopal, J., Huang, Z. M., Lim, C. T. and Ramakrishna, S. Biomacromolecules. 2005, 6, 2583.

[7] Tashiro, T. Macromol Mater Eng. 2001, 286, 63.

[8] Ignatova, M., Manolova, N. and Rashkov, I. Eur Polym J, 2007, 43, 1112.

[9] Teixeira, A. L., Abrams, G. A., Bertics, P.J., Murphy, C. J.and Nealey, P. F. J Cell Sci. 2003, 116, 1881.

[10] Venugopal, J., Low, S., Choon, A. T.and Ramakrishna, S. J Biomed Mater Res Part B Appl Biomater. 2008, 84B, 34.

[11] Mark, K., Park, J., Bauer, S. and Schmuki, P. Cell Tissue Res. 2010, 339, 131.

[12] Kim, S. K. Chitin, Chitosan, Oligosaccharides and Their Derivatives: Biological Activities and Applications, CRC Press, 2010; Part II, Chapter 8, pp 91.

[13] Kim, I. Y., Seo, S. J. and Moon, H. S. Biotechnology Advances. 2008, 26, 1.

[14] Muzzarelli, R. Carbohydr. Polym. 2009, 76, 167.

[15] Greiner, A.and Wendorff, J. H. Angew. Chem. Int. Ed. 2007, 46, 5670.

[16] Deitzel, J. M., Kleinmeyer, J. and Harris, D. Polymer. 2001, 42, 261.

[17] Veiseh, O., Sun, C., Fang, C., Bhattarai, N. and Gunn, J. Cancer Res. 2009, 69, 6200.

[18] Torres-Giner, S., Ocio, M. J. and Lagaron, J. M. Engineering in Life Sciences. 2008, 8, 303.

[19] Islam, M. S. and Karim, M. R. Colloids and Surfaces A: Physicochemical and Engineering Aspects. 2010, 366, 135.

[20] Jung, K. H., Huh, M. W., Yuan, J. and Hyun, S. H. Journal of Applied Polymer Science. 2007, 10, 2816.

[21] Cooper, A., Bhattarai, N., Kievit, M. F., Rossol, M. and Zhang, M. Q. Phys. Chem. Chem. Phys. 2011, 13, 9969.

[22] Puttipipatkhachorn, S., Nunthanid, J., Yamamoto, K. and Peck, G. E. J. Controlled Release. 2001, 75, 143. 